Book Review:

\title{
Code Breaker: Increase Creativity, Remix Assessment, and Develop a Class of Coder Ninjas!
}

by Brian Aspinall

San Diego, CA: Dave Burgess Publishing, 2017, 80 pages

ISBN: 978-1-946444-54-7 (Paperback)

\section{Reviewed by:}

Deanna Pecaski McLennan

Greater Essex County District School Board

There is a need for today's educators to better prepare children for life in an unknown future, integrating 21 st century competencies into daily work and inspiring children to engage in rich, creative problem solving in the classroom and beyond. In Brian Aspinall's book Code Breaker: Increase Creativity, Remix Assessment, and Develop a Class of Coder Ninjas, readers are taken on a journey through the author's experiences using authentic, student-centred activities and challenged to rethink their definition of what computational thinking is, and its application to today's curriculum. Computational thinking encompasses so much more than tech skills; it requires students to be passionate, creative and independent risk-takers who are not scared to stand alone in their thinking and communicate their ideas in non-traditional ways. Drawing upon the research of thinkers such as Piaget, Papert, and Wing, Aspinall advocates for the promotion of growth mindset in the classroom where both educators and children are encouraged to make mistakes and embrace uncertainty in their learning every day.

In his writing, Aspinall shares how his early teaching experiences ultimately shaped who he became as an educator. He challenges readers to redefine their roles, abandoning comfortable practices and embracing change and student led learning in their lessons. It can sometimes feel intimidating for educators to take risks and try something new. Aspinall weaves the voices and experiences of former students and colleagues throughout the text, using these to provide an authentic voice to the argument he is making for promoting change and growth mindset in the classroom. These points are also illuminated by meaningful examples from Aspinall's career, stories of how coding and computational thinking became vehicles for learning and change in his 
practice, helping transforming ordinary lesson plans into differentiated models that met greater student interest and need.

This book is divided into three main parts. The first introduces us to Aspinall as both a university student and educator, sharing how his early experiences studying computer programming and theories of computational thinking helped him later adapt his grade eight classroom to better respond to children's emerging interests during regular lessons. After delving into the work of many respected theorists, readers are able to enhance their understanding of what computational thinking is, including the language of coding, and how it can help teachers transform not only their classrooms but also their sense of self and identity as educators. In the final section of the book, practical examples of how computational thinking and coding have been used in a variety of grades and subject areas are shared, providing the reader with tangible evidence of how others have used these to integrate subjects in the classroom, engage children in complex thinking, and rethink traditional assessment practices. Many of these can be easily replicated. These activities have multiple entry points for all children, and can be used to differentiate regular classroom content for all learners.

As a strong advocate for technology embedded learning, Aspinall reassures readers that they do not have to be perfect educators, or proficient coders, in order to create change in their classrooms. Encouraging children to be creative and critically empowered learners who help shape the culture of the classroom is what matters. Readers do not require experience or comfort using technology or coding in order to glean many ideas for immediate use in their classrooms. In fact, Aspinall argues this point by including various educational missteps that he's had in his career, using these as opportunities to learn and grow as an educator and modelling a positive mindset for students.

Code Breaker is a fun, introductory text that inspires educators, administrators, coaches, and educational stakeholders to consider the various ways tech can enhance their programs as they become coders together with their students. Doing so can help them break free of rote, traditional practices, empower their students to take charge of their own learning, and begin to change the world. Aspinall reminds us that there is no right or wrong way to change your practice - the main thing is simply to just start. 